\title{
OS PERIGOS POR TRÁS DOS PRODUTOS QUÍMICOS DESTINADOS À ESTÉTICA CAPILAR: ANÁLISE DE FORMALDEÍDO
}

\section{THE HAZARDS ABOUT CHEMICALS INTENDED FOR HAIR AESTHETICS: FORMALDEHYDE ANALYSIS}

Daniel Ângelo Macena; Weller Jean Bérgamo; Patrícia Alexandra Antunes.

Universidade do Oeste Paulista - UNOESTE, Faculdade de Artes, Ciências, Letras e Educação de Presidente Prudente - FACLEPP

e-mail: daniel@unoeste.br

RESUMO - Com o intuito de melhor resultado em técnicas de alisamento capilar, ainda acontece a adição de formaldeído na composição do produto comercial aumentando os riscos de intoxicação e câncer no usuário. O objetivo do estudo foi avaliar quali-quantitativamente a concentração de formaldeído em alisantes capilar. Foi utilizado meio ácido e reagente de Schiff para identificação e na avaliação quantitativa espectrofotometria visível e aplicação de questionário as clientes. Três amostras apresentou-se fora dos padrões que a Anvisa estabelece, por não ser permitido uso acima de 0,2\% de Formaldeído no produto, na análise das questões percebeu-se que $50 \%$ das mulheres entrevistadas utilizam este tipo de tratamento, mesmo sabendo dos sintomas, riscos e efeitos posterior não desejáveis e gastam de $R \$ 100,00$ a $R \$ 150,00$ mensalmente no salão. Concluímos que o uso do formaldeído ainda é uma pratica comum, mesmo com tantos perigos e ser proibido pelo que estabelece a Anvisa.

Palavras-chave: Cabelo. Tratamento capilar. Análise de formaldeído. Efeitos tóxicos.

ABSTRACT - In order to achieve a better result in capillary smoothing techniques, the addition of Formaldehyde in the composition of the commercial product increases the risks of intoxication and cancer in the user. The objective of the study was to quali-quantitatively evaluate the concentration of formaldehyde in capillary straighteners. Acid medium and Schiff's reagent were used for identification and quantitative evaluation of visible spectrophotometry and questionnaire application to clients. Three samples were out of the standards that Anvisa establishes, because it is not allowed to use above $0.2 \%$ of formaldehyde in the product, in the analysis of the questions it was noticed that $50 \%$ of the interviewed women use this type of treatment, even knowing of the symptoms, risks and after effects not desirable and spend from $\mathrm{R} \$ 100$ to $\mathrm{R} \$ 150$ a month in the salon. We conclude that the use of formaldehyde is still a common practice, even with so many hazards and be prohibited by what establishes Anvisa.

Keywords: Hair. Hair treatment. Formaldehyde analysis. Toxic effects. 


\section{INTRODUÇÃO}

Se utilizado inadequadamente ou sem o auxílio de um profissional, os alisantes podem causar efeitos adversos como: queimaduras graves na córnea e no couro cabeludo, quebra dos fios e queda dos cabelos, já que esses produtos podem conter substâncias potencialmente tóxicas e/ou irritantes à pele e a saúde. Entretanto, quando os produtos atendem às exigências estabelecidas na legislação sanitária e sua utilização é feita de forma adequada, os danos à saúde são minimizados ou não ocasionados (MACAGNAN et al., 2017).

Desta forma, a Anvisa que coordena o Sistema Nacional de Vigilância Sanitária (SNVS), encontra-se vinculada ao Ministério da Saúde e integra o Sistema Único de Saúde (SUS) adotando princípios e diretrizes (SOUSA, 2017). Consequentemente, os alisantes devem, obrigatoriamente, ser registrados junto a Anvisa, para evitar riscos ou adulterações dos produtos comerciais registrado legalmente (ANVISA $A_{a}, 2005$; $\left.\mathrm{ANVISA}_{b}, 2005\right)$. De maneira geral, os alisantes químicos agem de forma muito similar aos agentes onduladores permanentes, são agentes químicos que rompem as ligações dissulfeto da queratina dos cabelos, permitindo a reestruturação do fio (DELFINI, 2011).

Dentre os alisantes químicos, está presente o Formol, que é uma solução aquosa de Formaldeído a 35\%, contendo metanol como preservativo contra a polimerização (MENDES, 1995). É o mais abundante e importante aldeído no ambiente de odor forte e irritante, muito solúvel em água, produzindo um hidrato com alta reatividade química (NACIONAL RESEARCH COUNCIL. 1981). O Formaldeído liga-se às proteínas da cutícula e aos aminoácidos da solução queratinizada dando origem a um "filme endurecedor" ao Longo do fio, com isso, impermeabiliza o mesmo e o mantém rígido e liso (SPAGNOL ABRAHAM, 2009, p 178-185). Mesmo sendo largamente utilizado e eficiente no resultado final em métodos de alisamento, como na escova progressiva, o Formol não é autorizado pela Anvisa para esse fim, pois é um composto cancerígeno pela Organização Mundial da Saúde (BELVISO, 2015).

A propósito, existem cremes alisantes comerciais que são alterados com a adição de formaldeído, provocando consequentemente, alterações no produto. Estes produtos têm a finalidade de alisar os cabelos mais que o normal mesmo sabendo que seu uso é proibido. O Formol é permitido somente como conservante dos produtos capilares em concentração máxima de $0,2 \%$, conforme a Resolução 162/01 e como agente endurecedor de unhas com limite máximo de uso permitido de $5 \%$ de acordo com a Resolução 215/05). Não é permitido qualquer concentração adicional que não seja do fabricante para fins de alisamento capilar.

No entanto, o objetivo deste estudo foi avaliar quali-quantitativamente a concentração de formaldeído em produtos para tratamento capilar utilizados em dois salões na cidade de lacri-SP e investigar através de questionário o conhecimento sobre os perigos, causas e consequências ao utilizar Formaldeído.

\section{MATERIAL E MÉTODOS}

As amostras dos produtos para alisamento capilar foram obtidas em dois salões de tratamento capilar em lacri, uma cidade do interior de São Paulo e avaliadas no laboratório de Química Farmacêutica da Universidade do Oeste Paulista (UNOESTE), totalizando quatro diferentes amostras de produtos iguais que são utilizadas nos dois diferentes salões.

A etapa analítica foi subdividida em duas partes, sendo a primeira um ensaio qualitativo, no qual o princípio do método é a formação do Formaldeído livre e combinado, em meio sulfúrico, na presença do reagente de Schiff (MORITA; ASSUMPÇÃO, 1988), quando positivo, indica uma coloração rósea. Na segunda parte, o princípio do método para análise quantitativa consiste na reação do Formaldeído com acetilacetona em presença do acetato de amônio para formar o 3,5-diacetil-1,4-dihidrolutidina.

A análise quantitativa de Formaldeído foi baseada no 'Dosamento Global de Formaldeído por Colorimetria com Acetilacetona aplicada para a análise da concentração de formol em produtos químicos destinados à estética capilar, conforme proposto pela ANVISA no Guia de Controle de Qualidade de Produtos Cosméticos (ANVISA 2005), na qual, a técnica analítica utilizada para a determinação do teor de Formaldeído nos produtos capilares foi espectrofotometria visível, utilizando espectrofotômetro da marca Quimis modelo Q7980P.

O Formaldeído foi extraído com 1butanol e realizada a leitura da absorbância com auxílio do espectrofotômetro visível a $410 \mathrm{~nm}$. Nesta etapa preparou-se uma curva padrão com 
cinco pontos de concentração conhecida de Formaldeído (0,019; 0,038; 0,056; 0,075; e 0,094 mg. $\left.L^{-1}\right)$.

Em uma segunda etapa foi aplicado um questionário a 19 usuários dos salões onde foram coletadas as amostras. As questões envolviam dados pessoais, frequência dos tratamentos capilares e irritabilidade dos produtos utilizados. O questionário foi aplicado após a assinatura do Termo Consentimento Livre e Esclarecido, aprovado previamente pelo Comitê de Ética de Pesquisa da Universidade sob número de protocolo 21009313.2.0000.5515.

\section{RESULTADOS E DISCUSSÃO}

\subsection{Análises Quali e Quantitativa}

Os ensaios analíticos foram iniciados pelo método qualitativo para verificar a presença ou não Formaldeído nas amostras coletadas. A Figura 1 apresenta as amostras em suas colorações originais e após a aplicação do ensaio analítico. A coloração surge após a adição do ácido e do reagente de Schiff. A intensidade da coloração rósea é indicada de acordo com a concentração do formaldeído presente no produto.

Figura 1. Coloração das amostras originais e depois da aplicação do reagente de Schiff.

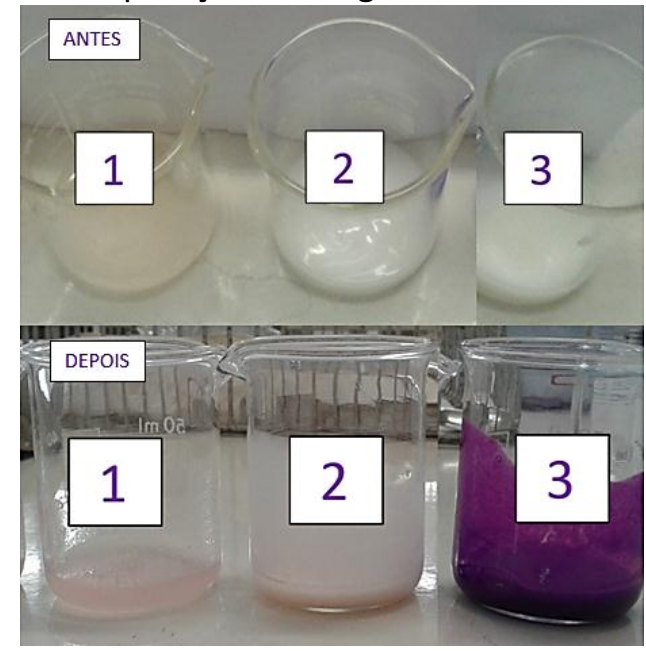

Fonte: Próprio autor

A quarta amostra não foi possível identificar e quantificar Formaldeído, devido a coloração própria (roxa) apresentada, pois tratase de um produto voltado diretamente para cabelos loiros o que justifica a coloração advinda de fábrica, já que são produtos específicos. Nas outras amostras analisadas (Figura 1), é possível perceber a presença de formaldeído, principalmente na amostra três, que apresentou uma coloração rósea de alta intensidade.

$\mathrm{Na}$ análise quantitativa, por meio da regressão linear dos pontos, obteve-se a equação da reta (Figura 2), e através da mesma foi possível calcular a concentração de formaldeído em cada amostra analisada. Os valores obtidos foram compilados e apresentam-se na Tabela 1.

Figura 2.- Curva analítica padrão de Formaldeído.

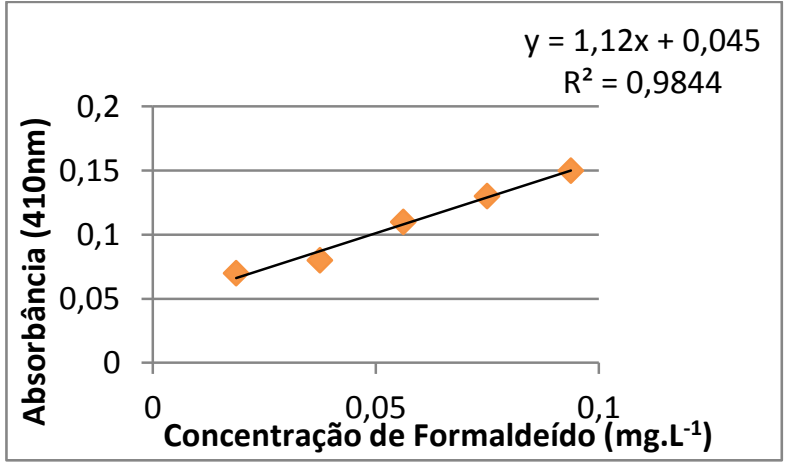

Fonte: Dados experimentais

Tabela 1. Médias do resultados analíticos quantitativos.

\begin{tabular}{ccc} 
Amostra & $\begin{array}{c}\text { Média } \\
\text { Concentração } \\
\text { (Convertido em \%) }\end{array}$ & $\begin{array}{c}\text { Absorbância } \\
\mathbf{( 4 1 0 ~} \mathbf{~ n m )}\end{array}$ \\
\hline $\mathbf{1}$ & 1,31 & 0,147 \\
\hline $\mathbf{2}$ & 1,34 & 0,150 \\
\hline $\mathbf{3}$ & ND* $^{*}$ & ND* $^{*}$ \\
\hline
\end{tabular}

Fonte: Dados experimentais.

Nota: ND = Não Detectável.

Comparando os resultados analíticos qualitativos com os resultados analíticos quantitativos após leitura em espectrofotômetro a $410 \mathrm{~nm}$, fica claro que as intensidades das amostras identificando a presença de Formaldeído estão condizentes na Tabela 1. Devido à alta intensidade rósea da amostra 3 não foi possível detectar a quantidade de Formaldeído no produto, pois a amostra apresentou-se acima do limite máximo de quantificação pelo equipamento. Conforme a Resolução 162/01 que permite a concentração de $0,2 \%$ de Formaldeído como conservantes, podemos então concluir que as três amostras analisadas apresentaram-se acima do valor máximo permitido, salvo a quarta amostra que não se pode ter certeza da presença ou não de Formaldeído em sua composição. 


\subsection{Questionário}

O questionário foi respondido por 19 indivíduos do sexo feminino que frequentam os salões de beleza, sendo que $3 \%$ possui menos de 20 anos, $32 \%$ entre 20 e 25 anos, $32 \%$ entre 26 e 35 e $32 \%$ possuem mais de 35 anos. Os resultados foram compilados e estão representados na Figura 3.

Em relação a utilização dos serviços do salão, mais de $45 \%$ das mulheres disseram manter o tratamento em dia, indo ao salão entre uma a duas vezes por mês, enquanto que a média de $35 \%$ das entrevistadas disseram que os cuidados uma a duas vezes ao ano é suficiente. Mais de $50 \%$ confirma que os gastos mensais no salão de beleza fica entre $\mathrm{R} \$ 100,00$ e $\mathrm{R} \$ 150,00$; enquanto que $20 \%$ delas não se importam em gastar mais chegando a ser acima de $\mathrm{R} \$ 250,00$.

As diferenças nos valores gastos estão diretamente relacionadas com os tipos de tratamento que costumam utilizar. Questionadas quanto ao tipo de tratamento mais utilizados, aproximadamente $45 \%$ fazem uso de técnicas rápidas, onde o princípio da técnica é o uso apenas do secador de cabelo e/ou prancha alisadora, tal qual, escova ou chapinha. Enquanto que mais de $50 \%$ prefere o alisamento permanente, pela durabilidade no resultado e pela praticidade do dia a dia segundo elas.

A maioria das entrevistadas tem cabelos ondulados e cacheados, porém no quesito tipo de cabelo mais de $70 \%$ são insatisfeitas com os seus cabelos e disseram que se pudesse escolheria ter cabelos lisos e aproximadamente $30 \%$ gostaria de ter cabelos cacheados. Nenhuma das entrevistadas gostaria de ter cabelos crespos/afro ou ondulados.

Apesar da satisfação positiva das clientes quanto ao resultado final, existem alguns efeitos a curto e longo prazo que foram confirmado pelas próprias usuárias dos serviços de alisamento. A maioria sentem no momento em que é aplicado o produto, principalmente, irritação no nariz, olhos lacrimejando e ardência no couro cabeludo, efeitos colaterais característicos que são causados pelo Formaldeído quando utilizado. 
Figura 3. Avaliação dos usuários, segundo utilização, gastos, tratamentos e tipos de cabelos (Figura 1a; 1b, 1c; 1d), e causas, efeitos a curto e longo prazo (Figura 1e; 1f; $1 \mathrm{~g}$ ).

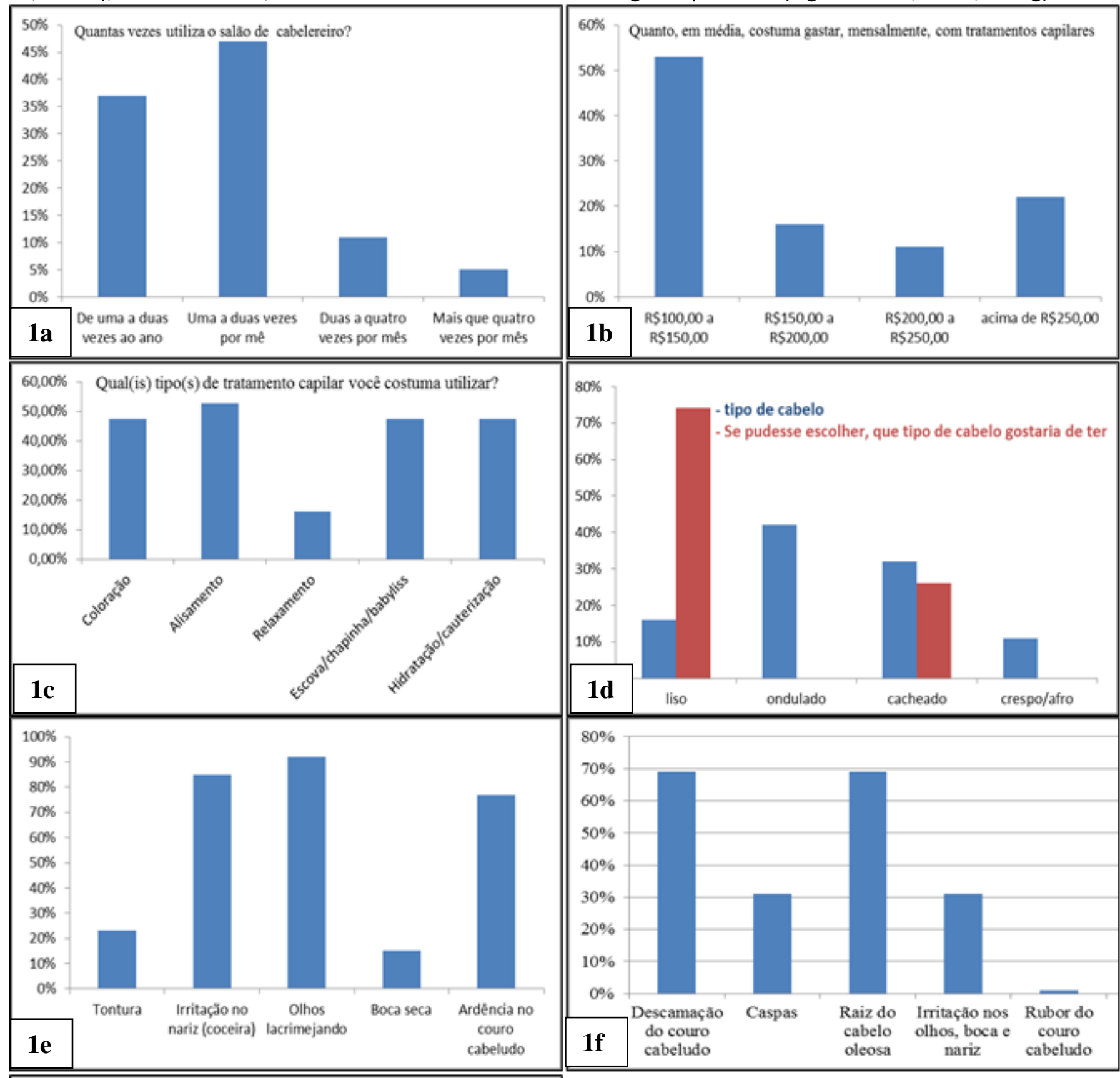

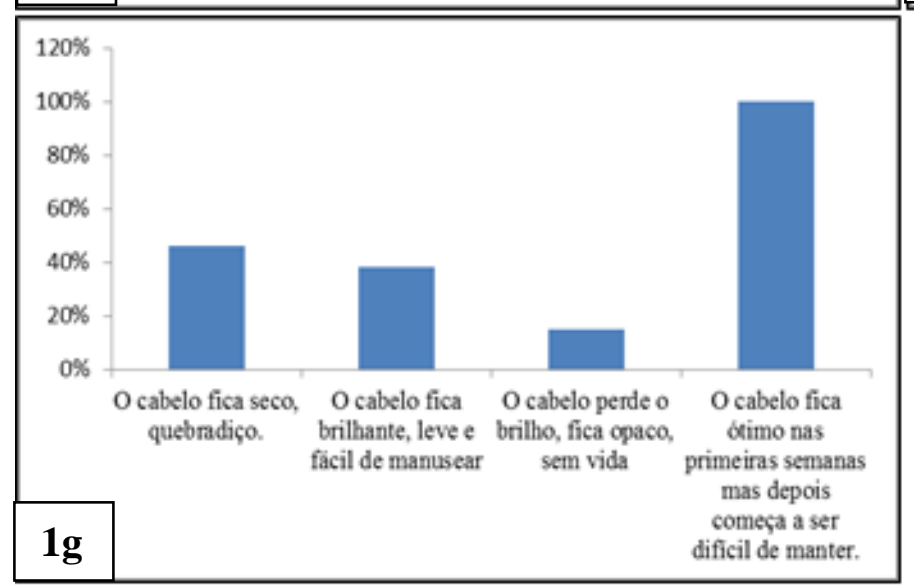


Já a longo prazo queixaram-se da descamação do couro cabeludo e oleosidade do cabelo. Concluíram que procuram pelos serviços novamente porque nas primeiras semanas fica ótimo, mas depois é difícil manter.

\section{CONSIDERAÇÕES FINAIS}

Sabe-se dos efeitos colaterais e consequências a curto e longo prazo causados pelo alisamento, porém, é o tratamento mais procurado nos salões de beleza. $\mathrm{O}$ grande risco que está atrelado a este tipo de serviço, tanto para os profissionais, quanto para os usuários, é quando os produtos são adulterados e contém uma quantidade maior de Formaldeído em sua composição, como foi confirmado nos resultados de três diferentes amostras neste trabalho. Isso torna suscetível de problemas graves futuros, como câncer por exemplo, já que é considerado carcinogênico.

Conclui-se que cabe mais atenção dos órgãos fiscalizadores em salões de beleza que prestam serviços voltados para estética capilar, a fim de certificar-se que os produtos são seguros devidamente seguros, se são adulterados pelo fabricante ou pelos próprios profissionais.

\section{REFERÊNCIAS}

ANVISA $_{a}$ - Agência Nacional de Vigilância Sanitária. Resolução RDC n. 211, 14 de julho de 2005. Define e classifica os produtos de higiene pessoal, cosméticos e perfumes em seu grau de risco. Diário Oficial [da] República Federativa do Brasil. Poder Executivo, Brasília, DF, 18 jul. 2005. Seção 1, p.58-60

ANVISA $_{b}$ - Agência Nacional de Vigilância Sanitária. Resolução RDC n. 215, de 26 de julho de 2005. Estabelece a lista de substâncias que os produtos de higiene pessoal, cosméticos e perfumes não devem conter, exceto nas condições e com as restrições estabelecidas. Diário Oficial [da] República Federativa do Brasil. Poder Executivo, Brasília, DF, 26 jul 2005. Seção 1, p.22-7.

BELVISO, T. I. Os perigos do uso inadequado do formol na estética capilar. Revista Intertox de Toxicologia, Risco Ambiental e Sociedade, v. 4, n. 1, 2015.
DELFINI, F. N. A. Ativos alisantes em cosméticos. 2011. 47 f. Trabalho de conclusão de curso (Farmácia-Bioquímica) - Universidade Estadual Paulista, Faculdade de Ciências Farmacêuticas de Araraquara, 2011.

MACAGNAN, K. K.; SARTORI, M. R. K.; CASTRO, F. $G$. Sinais e sintomas da toxidade do formaldeído em usuários de produtos alisantes capilares. Cadernos da Escola de Saúde, v. 2, n. 4, 2017.

MENDES, R. Patologia do trabalho. In: Patologia do trabalho. Atheneu, 1995.

MORITA, T.; ASSUMPÇÃO, R. M. V. Manual de Soluções, Reagentes e Solventes: Padronização, Preparação, Purificação. 20 ed. 6응 reimpressão. Edgar Blucher Ltda, 1988.

NATIONAL RESEARCH COUNCIL. Formaldehyde and other aldehydes. National Academies, 1981.

Resolução - RDC no 162 de 11 de setembro de 2001. Estabelece a Lista de Substancias de Ação Conservante para Produtos de Higiene Pessoal, Cosmético e Perfumes. Brasília: Diário Oficial da União; seção 1; n. 189; 02-10-2009; p. 58-60.

SOUSA, L. C. M. A vigilância em saúde sob a perspectiva de seus trabalhadores. 2017. Universidade Federal do Rio Grande do Norte. Mestrado em Saúde Coletiva (Dissertação). Brasil.

SPAGNOL ABRAHAM, L., MATEUS MOREIRA, A., HANAUER DE MOURA, L., REIS GAVAZZONI DIAS, M. F., \& ALVIM SANT'ANNA ADDOR, F. Tratamentos estéticos e cuidados dos cabelos: uma visão médica (parte 2). Surgical \& Cosmetic Dermatology, v. 1, n. 4, 2009.

STAMPACCHIO, H. A Essência do Cabelo Perfeito. Editora Baraúna, 2010. 